\title{
Loneliness and multiple health domains: associations among emerging adults
}

\author{
Lilian G. Perez ${ }^{1}$ (D) Daniel Siconolfi ${ }^{2} \cdot$ Wendy M. Troxel $^{2} \cdot$ Joan S. Tucker $^{1}$ • \\ Rachana Seelam $^{1} \cdot$ Anthony Rodriguez $^{3} \cdot$ Regina A. Shih $^{4} \cdot$ Elizabeth J. D'Amico $^{1}$
}

Received: 28 May 2021 / Accepted: 19 November 2021 / Published online: 4 January 2022

(C) The Author(s), under exclusive licence to Springer Science+Business Media, LLC, part of Springer Nature 2021

\begin{abstract}
Emerging adults (18-25 years), particularly racially/ethnically diverse and sexual and gender minority populations, may experience loneliness following major life transitions. How loneliness relates to health and health disparities during this developmental period is not well understood. We examine associations of loneliness with physical (self-rated health), behavioral (alcohol/marijuana consequences; nicotine dependence), and health behavior outcomes (weekday and weekend sleep; trouble sleeping), and investigate moderating effects by sex, race/ethnicity, and sexual/gender minority (SGM) status. Adjusted models using cross-sectional data from 2,534 emerging adults, predominantly in California, examined associations between loneliness and each outcome and tested interactions of loneliness with sex, race/ethnicity, and SGM status. Higher loneliness was significantly associated with worse self-rated health, higher marijuana consequences, less weekday sleep, and greater odds of feeling bothered by trouble sleeping. None of the interactions were significant. Findings suggest that interventions to reduce loneliness may help
\end{abstract}

Supplementary Information The online version contains supplementary material available at https://doi.org/10.1007/ s10865-021-00267-1.

Lilian G. Perez lperez@rand.org

1 RAND Corporation, 1776 Main Street, PO Box 2138, Santa Monica, CA 90407-2138, USA

2 RAND Corporation, 4570 Fifth Avenue, Suite 600, Pittsburgh, PA 15213, USA

3 RAND Corporation, 20 Park Plaza, Suite 920, Boston, MA 02116, USA

4 RAND Corporation, 1200 South Hayes Street, Arlington, VA 22202, USA promote healthy development among emerging adults across subgroups.

Keywords Loneliness - Emerging adulthood Physical health $\cdot$ Substance use $\cdot$ Sleep $\cdot$ Effect modification

\section{Introduction}

One in five US adults reports often or always feeling lonely or socially isolated (DiJulio et al., 2018), and national household trends show declining marriage rates and more individuals living alone (US Census Bureau, 2018), pointing to an increasingly socially disconnected population. Research with middle-aged adults and older adults suggest that lack of social connection poses serious health risks (e.g., premature mortality) above other well-established risk factors such as smoking and obesity (Holt-Lunstad et al., 2010). Major shifts in social connections often occur earlier in life, particularly emerging adulthood (generally defined as age 18-25 years) as a result of multiple life transitions in education, employment, and relationships, among other areas (Lee et al., 2018), which can have significant implications for healthy development in adulthood. Life transitions may promote social health, such as a sense of belonging and membership in social groups, which are an important aspect of identity development in emerging adulthood (National Academies of Sciences, 2019); however, they can also present challenges such as increasing feelings of loneliness (von Soest et al., 2020). In the US, emerging adults report larger social networks than other age groups (Bruine de Bruin et al., 2020), yet they also report higher loneliness (Green et al., 2001). Loneliness-defined as the perceived deficiency of social relationships in terms of quantity (e.g., number of social contacts) or quality (e.g., closeness)—can 
exist despite the existence of a large social network, particularly if those contacts are not "connections" in the sense of social support, purpose, and meaning (Cacioppo et al., 2015). Loneliness is related to adverse health outcomes (Christiansen et al., 2020; Matthews et al., 2016; Richard et al., 2017), independent of other social health indicators such as social isolation (e.g., living alone) and social support (Cacioppo et al., 2015). However, there is limited understanding of how loneliness relates to emerging adults' health across different domains (physical, behavioral, and health behaviors).

Given that identity development is central to emerging adulthood, it is important to also understand how loneliness varies across population subgroups, particularly by sex, race/ ethnicity, or sexual/gender minority (SGM) status. Women, racial/ethnic minorities, and SGM populations often experience discrimination and stigmatization, which may negatively affect their social relationships, including increasing feelings of loneliness (Doyle \& Molix, 2016; Majeno et al., 2018). To date, research examining differences on loneliness by sex has produced equivocal findings. One meta-analysis of 575 studies found that males report slightly higher loneliness than females, but in more robust analyses, the overall effect was not significant (Maes et al., 2019). Fewer studies have assessed differences in loneliness by race/ethnicity or SGM status. For example, one study found higher levels of loneliness among those of Asian or "other" race/ethnicity compared to White young adults, (Lee et al., 2020) and another study found higher loneliness among individuals who identify as SGM compared to those who identify as cisgender/heterosexual (Doyle \& Molix, 2016). How these subgroups cope with loneliness can have important implications for health and tailoring of prevention programming. However, there is poor understanding of whether sex, race/ ethnicity, or SGM status moderate the relationship between loneliness and emerging adults' health.

Most studies on loneliness are with middle-aged or older adults and show that loneliness is linked to poor outcomes across the physical and behavioral health (mental health and substance use), and health behavior domains (Hawkley \& Cacioppo, 2010; Leigh-Hunt et al., 2017). Within the physical health domain, reviews show that loneliness is related to increased risk of chronic conditions, obesity, and poor self-rated health (Hawkley \& Cacioppo, 2010; LeighHunt et al., 2017; Petitte et al., 2015). Self-rated health is an important outcome to focus on because it peaks and then declines around age 21, particularly among males and Black individuals (Sokol et al., 2017). Two studies suggest that mental health (e.g., perceived stress and depression) is an important pathway by which loneliness contributes to negative health outcomes, including poor self-rated health (Goosby et al., 2013; Segrin \& Passalacqua, 2010). Such evidence has placed a spotlight on mental health as a key intervention target; however, understanding the role of loneliness, above and beyond mental health, is important for informing interventions aiming to improve social connections for better health and well-being. Further, longitudinal evidence shows that loneliness predicts depression, but not vice versa (Cacioppo et al., 2010), and that interventions targeting loneliness can help reduce depressive symptoms (VanderWeele et al., 2011). This suggests that loneliness and depression are distinct conditions whose contributions to health must be disentangled. However, few studies have examined how loneliness relates to self-rated physical health among emerging adults (Goosby et al., 2013; Richard et al., 2017), independent of mental health, and how this relationship varies across sex, racial/ethnic, or SGM subgroups.

Within the behavioral health domain, two cross-sectional studies with adults aged 18 to 35 years showed that loneliness was related to poor mental health (e.g., depression, stress) and substance use (e.g., alcohol, marijuana) (Horigian et al., 2020; Matthews et al., 2019). One study found that loneliness had an indirect association with higher drug and alcohol use severity (based on amount, frequency of use, and problems from using) via increases in depression and anxiety (Horigian et al., 2020). Evidence linking loneliness with problematic substance use such as experiencing problems from drug use or substance use dependence, independent of mental health, is critical for informing clinical interventions (D'Amico et al., 2019). Yet, there are significant gaps in understanding how loneliness is associated with other problematic substance use outcomes among emerging adults, such as problems or consequences from using marijuana or nicotine dependence from vaping. Furthermore, although research points to higher substance use in adult males (vs females) and Whites (vs other race/ethnicities) (Chen \& Jacobson, 2012), no study that we are aware of has examined how the relation between loneliness and problematic substance use varies across sex, racial/ethnic, or SGM subgroups.

Finally, in the health behavior domain, loneliness has been linked with several unhealthy behaviors such as physical inactivity, poor diet, and poor sleep (Hawkley \& Cacioppo, 2010; Leigh-Hunt et al., 2017; Matthews et al., 2017). Among these, sleep is a key behavior to focus on because poor sleep duration or quality is associated with numerous unhealthy behaviors (e.g., substance use, exercising less, overeating) and poor physical and mental health (Johnson \& Breslau, 2001; Owens et al., 2014). However, there is limited understanding of how loneliness may be related to sleep duration or sleep problems, independent of mental health, and whether such associations differ by sex, race/ethnicity, or SGM status.

To address these gaps, the primary aim of the present study is to examine associations of loneliness with health outcomes across the physical (self-rated health), behavioral 
Fig. 1 Conceptual framework of loneliness in relation to multiple health domains, independent of mental health and other confounders (Aim 1), and the potential moderating effects of socio-demographic factors (Aim 2)

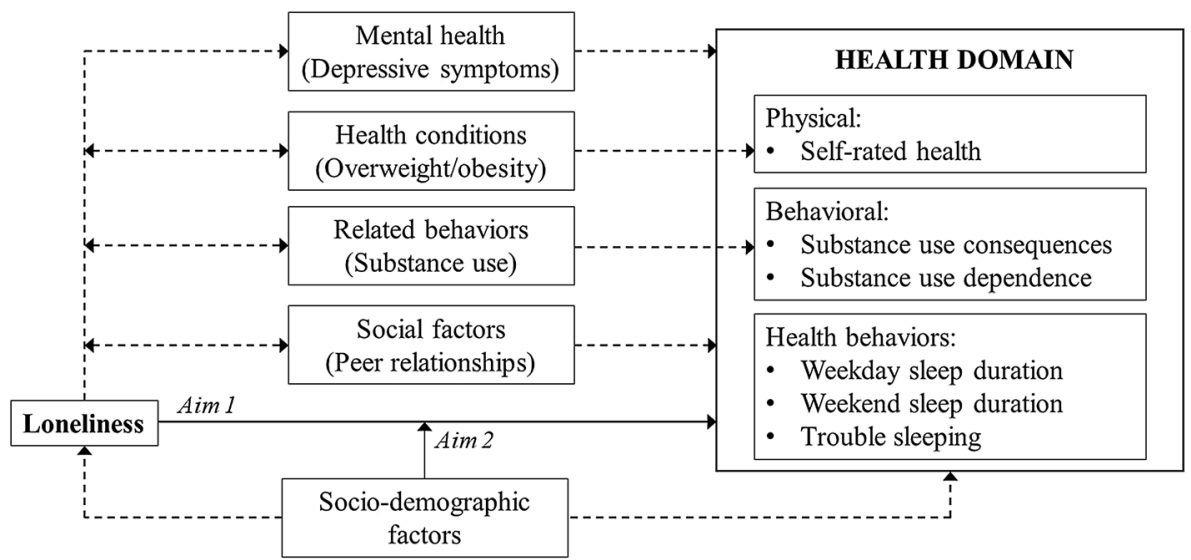

(substance use consequences and dependence), and health behavior domains (weekday and weekend sleep duration, and trouble sleeping), independent of mental health (i.e., depression) and other potential confounders, among a large and diverse sample of emerging adults (Fig. 1). The second aim is to investigate the moderating effects of sex, race/ethnicity, and SGM status on these associations.

\section{Methods}

Data come from a sample of emerging adults $(n=2,534$; mean age $=22.6$ years) who participated in an annual online survey from June 2019 to July 2020 (wave 12) as part of an ongoing longitudinal study. We focused on wave 12 when the loneliness measure was first introduced. About a quarter of the sample completed the survey during the COVID-19 pandemic (i.e., on or after stay-at-home orders were implemented on March 19, 2020); however, preliminary analyses showed no differences in the final results when accounting for the timing of survey completion. Thus, we performed all analyses without a COVID-19 timing covariate.

Participants were initially recruited in 2008 from 16 middle schools in southern California, when participants were in sixth or seventh grade (ages 11-13), for an evaluation of a voluntary after-school substance use prevention program (D'Amico et al., 2012). Parental informed consent and student assent were obtained. As participants transitioned from middle to high school, they were reconsented and asked to complete annual online surveys. Wave-to-wave retention rates average $85 \%$ across all 12 waves. Participants who do not complete a particular wave of data collection remain eligible to complete all subsequent waves. That is, they do not "drop out" of the study if they miss a survey wave; rather, we field the full sample at every wave so that all participants have an opportunity to participate in each survey. Substance use at wave 11 did not significantly predict retention at wave
12, similar to previous waves (D'Amico et al., 2018, 2020). However, at wave 12 , retention was higher among females (93.41\%) than males (90.53\%); Latinx/o (93.28\%) and Asian $(92.93 \%)$ participants were more likely to be retained than Whites $(90.87 \%)$, Blacks $(82.76 \%)$, or those of other race/ ethnicity $(90.11 \%)$; and those retained were slightly younger (mean age $=21.57$ years) than those who were not (mean age $=21.85$ ). Survey responses are protected by a Certificate of Confidentiality from the National Institutes of Health. Study procedures and materials were approved by the institution's internal review board.

\section{Measures}

\section{Loneliness}

The Three-Item Loneliness Scale, based on the UCLA Loneliness scale (Hughes et al., 2004), assessed how often participants felt they lacked companionship, felt left out, and felt isolated from others. Response options included: 1 = "hardly ever," $2=$ "some of the time," and 3= "often". Responses were summed to create a total loneliness score (range: $3-9$ ), with higher scores indicating greater loneliness.

\section{Outcomes}

\section{Self-reported health}

Participants were asked to rate their general health status using one item from the Short Form-12 health survey (Ware et al., 1996). Response options ranged from $1=$ "excellent" to $5=$ "poor" and were reverse coded; higher scores indicated better self-rated health.

\section{Substance use consequences and dependence}

Two outcomes focused on frequency of experiencing consequences from using marijuana or alcohol. We adapted 
items from three scales validated in emerging adult samples (Kahler et al., 2005; Simons et al., 2012; Tucker et al., 2003). We chose specific items from each scale that reflect a broad range of developmentally appropriate consequences, such as behavioral, psychological, or interpersonal problems. The marijuana and alcohol consequences items have been validated in other studies (Read et al., 2007; Simons et al., 2012) and showed high internal consistency in our sample (alpha $=0.87$ for marijuana consequences and 0.91 for alcohol consequences). Ten items assessed the number of times participants experienced specific problems from using marijuana (pot, weed, hash) in the past year (e.g., missed school, did something later regretted, got into trouble, trouble concentrating, trouble remembering, and relationships were negatively affected). Nine items assessed the number of times participants experienced specific problems from drinking alcohol (beer, wine, or hard liquor) in the past year (e.g., missed school/work, did something later regretted, got into trouble, felt really sick, and got into a fight or argument) (Kahler et al., 2005; Tucker et al., 2003). Response options for both the marijuana and alcohol consequences items included: $1=$ "never," $2=$ " 1 time," $3=$ " 2 times," $4=$ " $3-5$ times," $5=$ "6-9 times," $6=$ " $10-19$ times," and $7=$ " 20 or more times". Responses with ranges were recoded using the mid-point of the range (e.g., "3-5 times" was recoded as 4 times) and "20 or more times" was recoded as 20 . Separate sum scores were created for marijuana consequences and alcohol consequences, with higher scores indicating higher frequency of consequences from using the substance.

We also assessed nicotine dependence from cigarette/ tobacco use and vaping, separately. For nicotine dependence from cigarette/tobacco use (excluding vaping), participants were asked to report how often they experienced the following: intolerable craving if participant hasn't smoked for a few hours, reaching for cigarettes/tobacco without thinking about it, dropping everything to go out and buy cigarettes/ tobacco, and smoking/using more tobacco before going into a situation where smoking is prohibited (Shadel et al., 2014). The four items came from the PROMIS Nicotine Dependence Item Banks short form, which has been validated in daily and nondaily smokers (reliability $=0.81$ ) (Shadel et al., 2014). Similar items were used to assess nicotine dependence from vaping (Morean et al., 2019). Response options ranged from $0=$ "never" to $4=$ "almost always". Mean scores were computed for both dependence outcomes.

\section{Sleep duration and trouble sleeping}

We assessed three separate sleep outcomes: weekday sleep duration, weekend sleep duration, and trouble sleeping. Participants reported when they "usually go to bed" and "wake up" on the weekday ( 2 items) and weekend ( 2 items), similar to other studies (Larson et al., 2015; Pasch et al., 2010;
Troxel et al., 2019). The difference between bedtime and waketime was computed to obtain separate values for weekday and weekend sleep duration. We analyzed sleep during the weekday and weekend separately given variability in sleep times observed in emerging adults (i.e., later sleep times and rise times during the weekend) (Lund et al., 2010). Extreme values three or more standard deviations away from the mean for each variable were set to missing $(n=39$ participants for weekday sleep values; $n=33$ for weekend sleep values). We assessed trouble sleeping with one item from the Patient Health Questionnaire Somatic Symptom Severity Scale that asked how much participants were bothered by trouble sleeping in the past 4 weeks (Kroenke et al., 2002; Troxel et al., 2019). Response options included: $1=$ "not bothered at all," $2=$ "bothered a little," and $3=$ "bothered a lot".

\section{Hypothesized effect modifiers}

This analysis focused on three potential effect modifiers of associations between loneliness and health outcomes: sex, race/ethnicity, and SGM status. Participants reported their sex assigned at birth (male, female, or intersex/other). Only one participant reported being "intersex/other" and was dropped from the analyses. Participants also reported their race and ethnicity separately and were categorized into one of the following groups: White, Black, Latinx/o, Asian, American Indian or Alaska Native, Native Hawaiian or Pacific Islander, or multi-race. Few participants identified as American Indian/Alaska Native or Native Hawaiian/Pacific Islander $(n=7)$; thus, we combined them with those identifying as multi-racial $(n=120)$ to represent an "other" category. We created a composite measure for SGM status that captures both sexual behavior and sexual identity, which may not always be concordant (The GenIUSS Group, 2014). That is, participants were classified as SGM if they met any of the following criteria: reported a sexual orientation other than straight/heterosexual, reported being transgender, reported having sex with individuals of the same sex, reported a gender identity other than male or female, or if their current gender identity was different from their assigned sex at birth; else, they were classified as non-SGM.

\section{Covariates}

We controlled all models for variables significantly correlated with the outcomes in preliminary analyses, including socio-demographics, peer relationship functioning, and depressive symptoms. The self-rated health model additionally controlled for body mass index (BMI) given its significant correlation with this outcome $(\mathrm{r}=-0.27, p<0.0001)$ and observed relationship with loneliness in other studies 
(Lauder et al., 2006; Petitte et al., 2015). The substance use consequences and dependence outcomes additionally controlled for substance use.

\section{Socio-demographics}

Participants reported their age, sex, race/ethnicity, SGM status, highest education completed (high school diploma, GED or high school equivalent, and Bachelor's degree, trade school, etc.), and household composition (total number of different persons participant lives with such as husband or wife, parents, siblings, roommate, etc.).

\section{Peer relationship functioning}

We used the PROMIS ${ }^{\circledR}$ scale on peer relationship functioning, which assesses relationships with friends around social support, reciprocity, belongingness, and social participation (Dewalt et al., 2013). The scale asks the extent to which eight statements related to peer relationships are true in the past month: could count on friends, felt accepted by others, could talk about things with friends, others wanted to talk to me, others wanted to be with me, good at making friends, others wanted to be my friend, and friends and I helped each other out. Response options ranged from $1=$ "never" to $5=$ "always". Items were summed and transformed to a t-score, with higher values indicating better peer relationship functioning.

\section{Depressive symptoms}

The eight-item Patient Health Questionnaire assessed how often participants were bothered by a particular symptom, e.g., little interest or pleasure in doing things, over the past two weeks (Kroenke et al., 2009). Response options ranged from $1=$ "not at all" to $4=$ "nearly every day." Responses across items were summed, with higher scores indicating higher depressive symptoms.

\section{BMI status}

We estimated BMI $\left(\mathrm{kg} / \mathrm{m}^{2}\right)$ based on self-reported height and weight, and categorized participants as underweight or healthy weight $\left(\mathrm{BMI}<25 \mathrm{~kg} / \mathrm{m}^{2}\right)$ or overweight or obese $\left(25 \mathrm{~kg} / \mathrm{m}^{2} \leq \mathrm{BMI}\right)$.

\section{Substance use quantity}

We developed four separate items to assess quantity of substance participants used in a day: number of times marijuana was used (open-ended question); number of alcoholic drinks consumed, with response options ranging from "a few sips" (recoded to 0.25 drinks) to " 15 or more drinks" (recoded to $15)$; number of cigarettes smoked, with response options ranging from "less than 1 cigarette" (recoded to 0.5 cigarettes) to " 20 or more cigarettes (a pack or more)" (recoded to 20); and the number of times ("one time" defined as around 15 puffs or lasting around $10 \mathrm{~min}$ ) e-cigarettes were used (open-ended question).

\section{Analyses}

We computed descriptives (means or frequencies) for all eight outcomes, loneliness, and the covariate variables. We checked continuous outcomes for non-normality by examining the distributions, residuals, and skewness and kurtosis values. We also examined correlations among covariates to check for potential issues of multicollinearity. To address our first aim, we examined associations between loneliness and each outcome using linear regression for self-rated health and the two sleep duration outcomes; negative binomial models for the four substance use consequence and dependence outcomes, to account for their highly right-skewed distributions; and a multinomial model for the trouble sleeping outcome (reference: "not bothered at all"), similar to a previous study (Troxel et al., 2017) using these data. First, we ran bivariate models between loneliness and each outcome. Then, we adjusted models for the socio-demographic factors, depressive symptoms, and peer relationship functioning. BMI status was additionally included in the self-rated health model, and the quantity of substance use variables were included in their respective substance use consequence or dependence models (e.g., quantity of marijuana use was included in the marijuana consequences model). Given that problematic substance use may not be relevant to those who do not use the substance in question, we limited the consequence and dependence models to those who reported using marijuana or alcohol in the past year $(n=1234$ for marijuana consequences and $n=1937$ for alcohol consequences) and those who vaped nicotine or smoked cigarettes/used tobacco (excluding vaping) in the past month $(\mathrm{n}=404$ for dependence from vaping and $n=374$ for dependence from cigarettes/tobacco use). The timeframe (past year or month) was based on the wording of the items. Finally, to address our second aim, we tested separate models including two-way interactions of loneliness with sex, race/ethnicity, and SGM status (total of 3 interactions for each outcome). We used SAS v. 9.4 (SAS Institute Inc., Cary, NC) for all analyses. 
Table 1 Sample socio-demographic and health characteristics

\begin{tabular}{|c|c|}
\hline Characteristics & $\mathrm{n}(\%)$ or mean $(\mathrm{SD})$ \\
\hline \multicolumn{2}{|l|}{ Socio-demographics } \\
\hline Age (years), mean (SD) & $22.56(0.82)$ \\
\hline \multicolumn{2}{|l|}{ Sex, $\mathrm{n}(\%)$} \\
\hline Male & $1140(45.02)$ \\
\hline Female & $1392(54.98)$ \\
\hline \multicolumn{2}{|l|}{ Sexual/gender minority, n (\%) } \\
\hline No & $2089(82.47)$ \\
\hline Yes & $444(17.53)$ \\
\hline \multicolumn{2}{|l|}{ Race/ethnicity, n (\%) } \\
\hline White & $585(23.1)$ \\
\hline Black & $45(1.78)$ \\
\hline Latinx/o & $1184(46.74)$ \\
\hline Asian & $592(23.37)$ \\
\hline Other ${ }^{\mathrm{a}}$ & $127(5.01)$ \\
\hline \multicolumn{2}{|l|}{ Highest education completed, n (\%) } \\
\hline High school/GED & $1038(41.04)$ \\
\hline Post-secondary ${ }^{\mathrm{b}}$ & $1491(58.96)$ \\
\hline Household composition, mean $(\mathrm{SD})^{\mathrm{c}}$ & $1.33(0.70)$ \\
\hline \multicolumn{2}{|l|}{ Health outcomes } \\
\hline Self-rated health score, mean (SD) & $3.50(0.99)$ \\
\hline Marijuana consequences score, mean (SD) ${ }^{\mathrm{d}}$ & $10.68(22.64)$ \\
\hline Alcohol consequences score, mean $(\mathrm{SD})^{\mathrm{e}}$ & $8.02(16.86)$ \\
\hline Nicotine dependence from vaping score, mean $(S D)^{f}$ & $0.96(1.07)$ \\
\hline Nicotine dependence from cigarettes/tobacco use score, mean $(\mathrm{SD})^{\mathrm{g}}$ & $0.48(0.85)$ \\
\hline Weekday sleep (hours/day), mean (SD) & $7.82(1.40)$ \\
\hline Weekend sleep (hours/day), mean (SD) & $8.65(1.44)$ \\
\hline \multicolumn{2}{|l|}{ Trouble sleeping, $\mathrm{n}(\%)$} \\
\hline Not bothered at all & $1224(48.46)$ \\
\hline Bothered a little & $897(35.51)$ \\
\hline Bothered a lot & $405(16.03)$ \\
\hline \multicolumn{2}{|l|}{ Other covariates } \\
\hline Depressive symptoms, mean (SD) & $5.58(5.59)$ \\
\hline Peer relationship functioning score, mean (SD) & $50.00(10.00)$ \\
\hline \multicolumn{2}{|l|}{ Body mass index, $\mathrm{n}(\%)$} \\
\hline Underweight or healthy weight & $1370(56.49)$ \\
\hline Overweight/obese & $1055(43.51)$ \\
\hline Number of times used marijuana in a day, mean (SD) & $1.30(3.37)$ \\
\hline Number of alcoholic drinks consumed in a day, mean (SD) & $2.32(2.36)$ \\
\hline Number of times used e-cigarette in a day, mean (SD) & $1.16(5.24)$ \\
\hline Number of cigarettes smoked in a day, mean (SD) & $0.29(1.18)$ \\
\hline
\end{tabular}

${ }^{\mathrm{a}}$ Includes participants who identified as American Indian/Native Hawaiian $(\mathrm{n}=7)$ or multi-racial $(\mathrm{n}=120)$

bIncludes certificate programs, trade school, Bachelor's degree, or other advanced degree

${ }^{\mathrm{c}}$ Sum of different types of people living with (husband, wife, parents, roommate, etc.), range: 0 (alone)-5

${ }^{\mathrm{d}}$ Only among those who reported smoking marijuana in the past year, $\mathrm{n}=1234$

${ }^{\mathrm{e}}$ Only among those who reported drinking alcohol in the past year, $\mathrm{n}=1937$

${ }^{\mathrm{f}}$ Only among those who reported vaping e-cigarettes/personal vaporizers in the past month, $\mathrm{n}=404$

${ }^{g}$ Only among those who reported smoking cigarettes/using tobacco (excluding vaping) in the past month, $\mathrm{n}=374$ 
Table 2 Mean loneliness scores by sex, SGM status, race/ethnicity, and survey completion date

\begin{tabular}{lrcc}
\hline Characteristic & \multicolumn{1}{c}{$\mathrm{N}^{\mathrm{a}}$} & Mean (SD) & $p^{\mathrm{b}}$ \\
\hline Sex & & & 0.69 \\
$\quad$ Male & 1131 & $5.05(1.99)$ & \\
$\quad$ Female & 1382 & $5.02(1.94)$ & \\
Sexual/gender minority (SGM) & & & $<.0001$ \\
$\quad$ No & 2070 & $4.87(1.90)$ & \\
$\quad$ Yes & 443 & $5.81(2.05)$ & \\
Race/ethnicity & & & 0.63 \\
White & 581 & $5.04(1.92)$ & \\
Black & 45 & $5.36(2.31)$ & \\
Latinx/o & 1176 & $5.01(1.99)$ & \\
Asian & 586 & $5.09(1.90)$ & \\
$\quad$ Other & 125 & $4.90(2.05)$ & \\
Survey completion & & & 0.20 \\
Pre-pandemic & & & \\
Post-pandemic & 1882 & $5.06(1.97)$ & \\
\hline
\end{tabular}

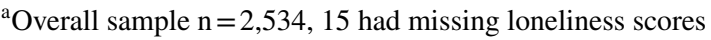

${ }^{\mathrm{b}}$ Based on ANOVA tests

${ }^{\mathrm{c}}$ Includes participants who identified as American Indian/Native Hawaiian $(n=7)$ or multi-racial $(n=120)$

${ }^{\mathrm{d}}$ Participant completed survey before (pre-pandemic) or on March 19, 2020 or later (post-pandemic)

\section{Results}

\section{Sample characteristics}

Table 1 presents socio-demographic and health characteristics of the sample. Participants were 22.6 years of age on average, with most completing some post-secondary education $(59 \%)$. Slightly over half were female, and $18 \%$ of the overall sample identified as SGM. The largest racial/ethnic group was Latinx/o (47\%), followed by White and Asian (23\% each); there were few Black participants (less than 2\%) and those of other race (5\%).

On average, the sample reported good overall health (score of 3.5 out of 5). Among those reporting marijuana use in the past year, the mean consequence score was approximately 10.7 (range: 0-200). Among those reporting alcohol use in the past year, the mean consequence score was approximately 8.0 (range: $0-180$ ). Scores were low for nicotine dependence from vaping (mean $=1.0$ out of a possible maximum of 4) and dependence from cigarette/tobacco use (mean $=0.5$ out of a possible maximum of 4 ). Average weekend sleep duration $(8.7 \mathrm{~h} /$ day $)$ was longer than weekday sleep ( $7.8 \mathrm{~h} /$ day). Overall, about half of the sample reported being bothered a little (36\%) or a lot (16\%) by trouble sleeping. The mean loneliness score was 5.0 (range: 3-9) and did not differ by sex, race/ethnicity, or survey completion

Table 3 Associations between loneliness and emerging adults' health outcomes across domains

\begin{tabular}{|c|c|c|c|c|}
\hline \multirow[t]{2}{*}{ Domain and outcomes } & \multicolumn{2}{|l|}{ Bivariate model $^{\mathrm{a}}$} & \multicolumn{2}{|l|}{ Adjusted model ${ }^{a, b}$} \\
\hline & $b(95 \% \mathrm{CI})$ & $p$ & $b(95 \% \mathrm{CI})$ & $p$ \\
\hline \multicolumn{5}{|l|}{ Physical } \\
\hline Self-rated health & $-0.15(-0.17,-0.13)$ & $<.0001$ & $-0.03(-0.05,-0.01)$ & 0.0086 \\
\hline \multicolumn{5}{|l|}{ Behavioral } \\
\hline Marijuana consequences & $0.20(0.13,0.26)$ & $<.0001$ & $0.10(0.02,0.17)$ & 0.0134 \\
\hline Alcohol consequences & $0.10(0.07,0.14)$ & $<.0001$ & $0.002(-0.04,0.04)$ & 0.9356 \\
\hline Nicotine dependence from vaping & $0.11(0.05,0.16)$ & $<.0001$ & $0.01(-0.05,0.08)$ & 0.7168 \\
\hline $\begin{array}{l}\text { Nicotine dependence from cigarette/ } \\
\text { tobacco use }\end{array}$ & $0.09(0.01,0.18)$ & 0.0331 & $-0.01(-0.10,0.09)$ & 0.5534 \\
\hline \multicolumn{5}{|l|}{ Health behavior } \\
\hline Weekday sleep duration & $-0.07(-0.10,-0.04)$ & $<.0001$ & $-0.04(-0.08,-0.01)$ & 0.0189 \\
\hline \multirow[t]{2}{*}{ Weekend sleep duration } & $-0.03(-0.06,-0.01)$ & 0.0190 & $-0.01(-0.04,0.03)$ & 0.7015 \\
\hline & OR $(95 \% \mathrm{CI})$ & $p$ & OR $(95 \% \mathrm{CI})$ & $p$ \\
\hline \multicolumn{5}{|l|}{ Trouble sleeping (ref: not bothered at all) } \\
\hline Bothered a little & $1.30(1.24,1.36)$ & $<.0001$ & $1.07(1.00,1.14)$ & 0.0405 \\
\hline Bothered a lot & $1.63(1.53,1.73)$ & $<.0001$ & $1.12(1.03,1.22)$ & 0.0095 \\
\hline
\end{tabular}

${ }^{a}$ Linear regression used for self-rated health and the sleep duration outcomes. Negative binomial models used for the consequences and dependence outcomes. Multinomial logistic regression used for trouble sleeping. All betas are unstandardized

${ }^{b}$ Adjusted for age, sex, race/ethnicity, sexual/gender minority status, education, household composition, depression, and peer relationship functioning; the self-rated health model is additionally adjusted for body mass index status and the consequences and dependence models are additionally adjusted for substance use quantity 
date (before or during the COVID-19 pandemic) (Table 2). SGM participants reported significantly higher loneliness $($ mean $=5.8)$ than their non-SGM peers $($ mean $=4.9)$, $p<0.0001$ (Table 2).

\section{Associations between loneliness and health}

Loneliness was significantly associated with worse health outcomes in all bivariate models (Table 3; correlations presented in Appendix A). After controlling for sociodemographics, depressive symptoms, peer relationship functioning, and other covariates, about half of the associations remained significant (Table 3; model building steps presented in Appendix B). The fully-adjusted models show that a 1 unit increase in loneliness was associated with a 0.03 unit decrease in self-rated health $(p=0.009) ; 0.10$ increase in the log number of marijuana consequences, i.e., 1.10 times higher count of marijuana consequences $(p=0.01)$; $0.04 \mathrm{~h} /$ day decrease in weekday sleep $(p=0.02)$; the odds of feeling bothered a little by trouble sleeping was 1.07 times greater than those not bothered at all $(p=0.04)$; and the odds of feeling bothered a lot by trouble sleeping was 1.12 times greater than those not bothered at all $(p=0.01)$ (Table 3$)$. The final set of models testing interactions of loneliness with sex, race/ethnicity, and SGM status showed that none of the interactions were significant at $p<0.05$ (Appendix C).

\section{Discussion}

The present study addresses gaps in our understanding of the association between loneliness and health across multiple domains among emerging adults, and whether these associations varied by sex, race/ethnicity, or SGM status. After controlling for depression and other potential confounders, we found small but significant associations of loneliness with worse self-rated health, more marijuana consequences, and poorer sleep (less weekday sleep and greater trouble sleeping). We did not find associations of loneliness with alcohol consequences, nicotine dependence from vaping or cigarette/ tobacco use, or weekend sleep duration. We also did not find significant differences across socio-demographic subgroups.

Overall, our sample of emerging adults reported loneliness scores that were slightly lower than that reported in a study of adults in their early $20 \mathrm{~s}$ who participated in a national web-based survey (Shovestul et al., 2020). Differences in loneliness scores between that study and our sample may be due in part to differences in socio-demographic (e.g., our sample was majority Latinx/o) and geographic characteristics (national vs. mainly California-based). Although there is evidence of increased loneliness among young adults during the COVID-19 pandemic (Bu et al., 2020; Horigian et al., 2020), we did not find differences in loneliness scores among those who completed the survey before versus during the pandemic. Given data collection for our survey ended in July 2020, it is possible that our data did not capture potential increases in loneliness as the pandemic continued throughout the year.

Similar to other work in this area with adolescents and adults, we found that loneliness was associated with worse self-rated health (Peltzer \& Pengpid, 2017; Richard et al., 2017; Segrin \& Passalacqua, 2010). One potential explanation for this is that loneliness is being driven by reduced social interactions with existing close contacts (e.g., family; high school friends) as emerging adults transition into new social roles such as college or new jobs. Reduced social interactions has been linked to greater physical symptom severity (Zhaoyang et al., 2019). Fewer interactions with parents may also mean that emerging adults experience reduced monitoring of their health behaviors, thereby creating a sense of freedom to engage in risky behaviors, such as substance use, which can contribute to poor physical health (He et al., 2004; Thompson et al., 2015).

For the substance use outcomes, our study is the first to show a link between loneliness and higher marijuana consequences in emerging adults. Other studies with this age group have shown positive associations between loneliness and frequency of marijuana use (Holmes et al., 2016; Rhew et al., 2021). Of note, our marijuana consequence model accounted for frequency of use, thus loneliness appears to have an independent association with marijuana consequences, which is suggestive of problematic use. One possible explanation for this finding is that individuals with higher levels of loneliness may use marijuana in riskier ways that contribute to more consequences, regardless of level of marijuana use. For example, one study showed that emerging adults who use marijuana to cope with negative affect (e.g., anger) or enhance the effects of other drugs are more likely to experience consequences, whereas those using it for experimental reasons experience fewer problems (Patrick et al., 2016). Further, lonely emerging adults may use more potent forms of marijuana to achieve their objectives, and potency is not captured in measures of marijuana use frequency. Our study did not assess potency of the marijuana products used or reasons for using marijuana, but future research is needed examining whether these factors explain the increased risk of marijuana consequences among those reporting higher loneliness despite levels of marijuana use. It is possible that someone who is experiencing many problems from using marijuana (e.g., missing school, doing something they later regretted) may also be more likely to become socially isolated and experience loneliness, pointing to a possible bi-directional relationship. Given the cross-sectional nature of this study, we cannot test the directionality of this association; however, this correlation highlights the importance of addressing loneliness 
during this developmental period. Additional studies are needed to replicate this finding and enhance understanding of the etiologic pathways.

Unexpectedly, we did not find significant associations of loneliness with alcohol consequences or nicotine dependence from cigarette/tobacco use or vaping, after adjusting for all covariates. A review of 25 studies found mixed evidence for the association between loneliness and smoking among adults and adolescents, with only half of the studies showing a significant association (Dyal \& Valente, 2015). The association between loneliness and substance use consequences or dependence may be complicated by other factors, which could account for these equivocal findings. For example, one study conducted during the COVID-19 pandemic found an indirect association between loneliness and higher alcohol and drug use severity, working through anxiety (Horigian et al., 2020). Although our survey assessed anxiety levels, we did not include it in the models given its high correlation with depression $(\mathrm{r}=0.78, p<0.0001)$ and lower correlations with our substance use and loneliness variables compared to the depression scale. Given the associations of loneliness with alcohol consequences and nicotine dependence from cigarette/tobacco or vaping were no longer significant with depression in the models, it is possible that depression is mediating these associations. Longitudinal data are needed to test these potential mediating pathways. Interestingly, the association between loneliness and marijuana consequences remained significant when we added depression and other covariates in the model, although the magnitude of the effect decreased. A possible explanation for the difference between the alcohol and marijuana consequences findings is that alcohol is more likely to be used for social purposes. Research shows that higher alcohol use is associated with better relationship functioning (D'Amico et al., 2016) and higher marijuana use is related to worse social well-being (Rhew et al., 2021). However, the association between loneliness and alcohol consequences became nonsignificant even before we controlled for peer relationship functioning, specifically when we adjusted for depressive symptoms. Thus, psychological rather than social factors appear to explain the association between loneliness and alcohol but not marijuana consequences in our sample.

With respect to the sleep outcomes, we found significant associations of loneliness with less weekday sleep duration and more trouble sleeping, but no association with weekend sleep duration. This is in line with findings from two other studies of adults (mean ages 18 or 21 years) showing loneliness is associated with worse sleep outcomes (Matthews et al., 2017; Peltzer \& Pengpid, 2017) and emphasizes the importance of measuring both weekday and weekend sleep. One potential explanation for why we found an association between loneliness and weekday sleep but not weekend sleep is that opportunities for socialization can vary throughout the week, and those opportunities may influence both feelings of loneliness and sleep/wake times. On weekdays, opportunities for socialization with close social ties may be reduced given work or school schedules. Stress related to work or school may in turn, contribute to trouble sleeping. In contrast, on weekends, there may be greater opportunities for socializing, and there is greater flexibility in terms of sleep/wake times. Thus, efforts to improve sleep during this developmental period could also target loneliness within the social contexts of weekdays (e.g., school or workplace).

There may also be a physiological explanation for our finding linking loneliness with less weekday sleep and trouble sleeping. For example, higher loneliness is associated with higher inflammatory markers (Cho et al., 2015; Smith et al., 2020), and inflammation can trigger changes in neurotransmitters that affect sleep patterns and behaviors (social withdrawal) (Irwin, 2019). A study of middle-aged adults found that inconsistent sleep is associated with higher levels of inflammatory markers (Dzierzewski et al., 2020). Thus, inflammation may lead to or be a consequence of poor sleep, and loneliness may exacerbate this process. Other physiological pathways, such as stress hormones (e.g., cortisol) associated with both sleep and loneliness may also play a role (Hawkley \& Cacioppo, 2010).

Interestingly, we did not find differences for the associations between loneliness and our outcomes by sex, race/ethnicity, or SGM status. Although we found greater loneliness among SGM compared to non-SGM participants, consistent with a study of adults (Doyle \& Molix, 2016), we did not find evidence of a higher risk of negative health outcomes in this subgroup. It is possible that the loneliness and health associations are moderated by other unmeasured structural factors that disproportionately affect women and minority populations, such as discrimination, rather than one's self-identity.

Our study has several limitations. First, our sample was limited to emerging adults largely residing in southern California; thus, findings cannot be generalized to the general emerging adult population. Second, although we had a large sample of Latinx/o participants, the proportion of Black participants was small (less than $2 \%$ ), which may have limited our ability to detect racial/ethnic moderating effects. Third, the cross-sectional design of this analysis does not allow us to make causal inferences. We discuss several potential psychosocial and physiological mechanisms by which loneliness may contribute to poor health, but longitudinal data are needed to test these hypotheses. Fourth, loneliness and our health outcomes were assessed by self-report, which are subject to respondent biases (e.g., recall, social desirability, same source). Nevertheless, some of the self-report measures used in our study have been shown to strongly correlate with their respective objective measures, e.g., sleep (Wolfson et al., 2003) and self-rated health (Wu et al., 
2013). Finally, although we controlled for peer relationship functioning and household composition, there may be other unmeasured social health indicators (e.g., social networks, social media use) that contribute to these associations.

\section{Conclusions}

This study used data from a diverse sample of emerging adults to understand the association of loneliness with health across physical, behavioral, and health behavior domains, and to test for differences in these associations by sex, race/ ethnicity, and SGM status. Overall, findings indicate that loneliness is associated with multiple adverse health outcomes in emerging adults, independent of other well-documented mental, social, and behavioral risk factors. Studies on the health effects of loneliness have largely focused on middle-aged and older adults; but high levels of loneliness in younger age groups highlights the need for more research on how loneliness may contribute to health across different domains during this important development period. Findings suggest that interventions to address loneliness among emerging adults are needed to promote health and positive development for this age group and potentially later in life as they transition to adulthood.

Acknowledgements The authors would like to thank the study participants, districts, and schools that participated in and supported the CHOICE project and Jennifer Parker for overseeing the web-based data collection.

Authors' contributions All authors contributed to the study conception and design. The first draft of the manuscript was written by LP and all authors commented on previous versions of the manuscript. Data analyses were performed by RS. All authors read and approved the final manuscript.

Funding This work was funded by grants from the National Institute on Alcohol Abuse and Alcoholism (R01AA016577, R01AA020883, R01AA025848; PI: D’Amico).

\section{Declarations}

Conflicts of interest The authors declare that they have no conflicts of interest.

Ethical approval All procedures were in accordance with the ethical standards of the RAND Human Subjects Protections Committee (institutional review board) and with the 1964 Helsinki declaration and its later amendments or comparable ethical standards.

Human and Animal Rights and Informed Consent All procedures followed were in accordance with ethical standards of the responsible committee on human experimentation (institutional and national) and with the Helsinki Declaration of 1975, as revised in 2000. Informed consent was obtained from all participants included in the study. All individual participants provided informed consent to participate and use of their data for publication.

\section{References}

Bruine de Bruin, W., Parker, A. M., \& Strough, J. (2020). Age differences in reported social networks and well-being. Psychology and Aging, 35, 159-168. https://doi.org/10.1037/pag0000415

Bu, F., Steptoe, A., \& Fancourt, D. (2020). Who is lonely in lockdown? Cross-cohort analyses of predictors of loneliness before and during the COVID-19 pandemic. Public Health, 186, 31-34. https:// doi.org/10.1016/j.puhe.2020.06.036

Cacioppo, J. T., Hawkley, L. C., \& Thisted, R. A. (2010). Perceived social isolation makes me sad: 5-year cross-lagged analyses of loneliness and depressive symptomatology in the Chicago health, aging, and social relations study. Psychology and Aging, 25, 453463. https://doi.org/10.1037/a0017216

Cacioppo, S., Grippo, A. J., London, S., Goossens, L., \& Cacioppo, J. T. (2015). Loneliness: Clinical import and interventions. Perspectives on Psychological Science, 10, 238-249. https://doi. org/10.1177/1745691615570616

Chen, P., \& Jacobson, K. C. (2012). Developmental trajectories of substance use from early adolescence to young adulthood: Gender and racial/ethnic differences. Journal of Adolescent Health, 50, 154-163. https://doi.org/10.1016/j.jadohealth.2011.05.013

Cho, H. J., Seeman, T. E., Kiefe, C. I., Lauderdale, D. S., \& Irwin, M. R. (2015). Sleep disturbance and longitudinal risk of inflammation: Moderating influences of social integration and social isolation in the Coronary Artery Risk Development in Young Adults (CARDIA) study. Brain, Behavior, and Immunity, 46, 319-326. https://doi.org/10.1016/j.bbi.2015.02.023

Christiansen, J., Lund, R., Qualter, P., Andersen, C. M., Pedersen, S. S., \& Lasgaard, M. (2020). Loneliness, social isolation, and chronic disease outcomes. Annals of Behavioral Medicine, 55, 203-215. https://doi.org/10.1093/abm/kaaa044

D’Amico, E. J., Parast, L., Osilla, K. C., Seelam, R., Meredith, L. S., Shadel, W. G., \& Stein, B. D. (2019). Understanding which teenagers benefit most from a brief primary care substance use intervention. Pediatrics, 144, e20183014. https://doi.org/10. 1542/peds.2018-3014

D’Amico, E. J., Rodriguez, A., Tucker, J. S., Dunbar, M. S., Pedersen, E. R., Shih, R. A., Davis, J. P., \& Seelam, R. (2020). Early and late adolescent factors that predict co-use of cannabis with alcohol and tobacco in young adulthood. Prevention Science, 21, 530-544. https://doi.org/10.1007/s11121-020-01086-7

D’Amico, E. J., Rodriguez, A., Tucker, J. S., Pedersen, E. R., \& Shih, R. A. (2018). Planting the seed for marijuana use: Changes in exposure to medical marijuana advertising and subsequent adolescent marijuana use, cognitions, and consequences over seven years. Drug and Alcohol Dependence, 188, 385-391. https://doi. org/10.1016/j.drugalcdep.2018.03.031

D’Amico, E. J., Tucker, J. S., Miles, J. N., Ewing, B. A., Shih, R. A., $\&$ Pedersen, E. R. (2016). Alcohol and marijuana use trajectories in a diverse longitudinal sample of adolescents: Examining use patterns from age 11 to 17 years. Addiction, 111, 18251835. https://doi.org/10.1111/add.13442

D’Amico, E. J., Tucker, J. S., Miles, J. N., Zhou, A. J., Shih, R. A., \& Green, H. D., Jr. (2012). Preventing alcohol use with a voluntary after-school program for middle school students: Results from a cluster randomized controlled trial of CHOICE. Prevention Science, 13, 415-425. https://doi.org/10.1007/s11121-011-0269-7

Dewalt, D. A., Thissen, D., Stucky, B. D., Langer, M. M., Morgan Dewitt, E., Irwin, D. E., Lai, J. S., Yeatts, K. B., Gross, H. 
E., Taylor, O., \& Varni, J. W. (2013). PROMIS Pediatric Peer Relationships Scale: Development of a peer relationships item bank as part of social health measurement. Health Psychology, 32, 1093-1103. https://doi.org/10.1037/a0032670

DiJulio, B., Hamel, L., Muñana, C., \& Brodie, M. (2018). Loneliness and social isolation in the United States, the United Kingdom, and Japan: An international survey. https://www.kff.org/other/ report/loneliness-and-social-isolation-in-the-united-states-theunited-kingdom-and-japan-an-international-survey/.

Doyle, D. M., \& Molix, L. (2016). Disparities in social health by sexual orientation and the etiologic role of self-reported discrimination. Archives of Sexual Behavior, 45, 1317-1327. https://doi. org/10.1007/s10508-015-0639-5

Dyal, S. R., \& Valente, T. W. (2015). A systematic review of loneliness and smoking: Small effects, big implications. Substance Use and Misuse, 50, 1697-1716. https://doi.org/10.3109/10826 084.2015.1027933

Dzierzewski, J. M., Donovan, E. K., Kay, D. B., Sannes, T. S., \& Bradbrook, K. E. (2020). Sleep inconsistency and markers of inflammation. Frontiers in Neurology, 11, 1042. https://doi.org/ 10.3389/fneur.2020.01042

Goosby, B. J., Bellatorre, A., Walsemann, K. M., \& Cheadle, J. E. (2013). Adolescent loneliness and health in early adulthood. Sociol Inquiry, 83. https://doi.org/10.1111/soin.12018

Green, L. R., Richardson, D. S., Lago, T., \& Schatten-Jones, E. C. (2001). Network correlates of social and emotional loneliness in young and older adults. Personality and Social Psychology Bulletin, 27, 281-288. https://doi.org/10.1177/0146167201273002

Hawkley, L. C., \& Cacioppo, J. T. (2010). Loneliness matters: A theoretical and empirical review of consequences and mechanisms. Annals of Behavioral Medicine, 40, 218-227. https://doi.org/10. 1007/s12160-010-9210-8

He, K., Kramer, E., Houser, R. F., Chomitz, V. R., \& Hacker, K. A. (2004). Defining and understanding healthy lifestyles choices for adolescents. Journal of Adolescent Health, 35, 26-33. https://doi. org/10.1016/j.jadohealth.2003.09.004

Holmes, L. M., Popova, L., \& Ling, P. M. (2016). State of transition: Marijuana use among young adults in the San Francisco Bay Area. Preventive Medicine, 90, 11-16. https://doi.org/10.1016/j.ypmed. 2016.06.025

Holt-Lunstad, J., Smith, T. B., \& Layton, J. B. (2010). Social relationships and mortality risk: A meta-analytic review. PLoS Medicine, 7, e1000316. https://doi.org/10.1371/journal.pmed.1000316

Horigian, V. E., Schmidt, R. D., \& Feaster, D. J. (2020). Loneliness, mental health, and substance use among US young adults during COVID-19. Journal of Psychoactive Drugs, 53, 1-9. https://doi. org/10.1080/02791072.2020.1836435

Hughes, M. E., Waite, L. J., Hawkley, L. C., \& Cacioppo, J. T. (2004). A short scale for measuring loneliness in large surveys: Results from two population-based studies. Research on Aging, 26, 655672. https://doi.org/10.1177/0164027504268574

Irwin, M. R. (2019). Sleep and inflammation: Partners in sickness and in health. Nature Reviews Immunology, 19, 702-715. https://doi. org/10.1038/s41577-019-0190-z

Johnson, E. O., \& Breslau, N. (2001). Sleep problems and substance use in adolescence. Drug and Alcohol Dependence, 64, 1-7. https://doi.org/10.1016/s0376-8716(00)00222-2

Kahler, C. W., Strong, D. R., \& Read, J. P. (2005). Toward efficient and comprehensive measurement of the alcohol problems continuum in college students: The brief young adult alcohol consequences questionnaire. Alcoholism, Clinical and Experimental Research, 29, 1180-1189. https://doi.org/10.1097/01.alc.00001 71940.95813.a5

Kroenke, K., Spitzer, R. L., \& Williams, J. B. (2002). The PHQ-15: Validity of a new measure for evaluating the severity of somatic symptoms. Psychosomatic Medicine, 64, 258-266. https://doi.org/ 10.1097/00006842-200203000-00008

Kroenke, K., Strine, T. W., Spitzer, R. L., Williams, J. B., Berry, J. T., \& Mokdad, A. H. (2009). The PHQ-8 as a measure of current depression in the general population. Journal of Affective Disorders, 114, 163-173. https://doi.org/10.1016/j.jad.2008.06.026

Larson, N., Laska, M. N., Story, M., \& Neumark-Sztainer, D. (2015). Sports and energy drink consumption are linked to health-risk behaviours among young adults. Public Health Nutrition, 18, 2794-2803. https://doi.org/10.1017/S1368980015000191

Lauder, W., Mummery, K., Jones, M., \& Caperchione, C. (2006). A comparison of health behaviours in lonely and non-lonely populations. Psychology, Health \& Medicine, 11, 233-245. https://doi. org/10.1080/13548500500266607

Lee, C. M., Cadigan, J. M., \& Rhew, I. C. (2020). Increases in loneliness among young adults during the COVID-19 pandemic and association with increases in mental health problems. Journal of Adolescent Health, 67, 714-717. https://doi.org/10.1016/j.jadoh ealth.2020.08.009

Lee, T. K., Wickrama, K. A. S., O’Neal, C. W., \& Prado, G. (2018). Identifying diverse life transition patterns from adolescence to young adulthood: The influence of early socioeconomic context. Social Science Research, 70, 212-228. https://doi.org/10.1016/j. ssresearch.2017.12.001

Leigh-Hunt, N., Bagguley, D., Bash, K., Turner, V., Turnbull, S., Valtorta, N., \& Caan, W. (2017). An overview of systematic reviews on the public health consequences of social isolation and loneliness. Public Health, 152, 157-171. https://doi.org/10.1016/j.puhe. 2017.07.035

Lund, H. G., Reider, B. D., Whiting, A. B., \& Prichard, J. R. (2010). Sleep patterns and predictors of disturbed sleep in a large population of college students. Journal of Adolescent Health, 46, 124 132. https://doi.org/10.1016/j.jadohealth.2009.06.016

Maes, M., Qualter, P., Vanhalst, J., Van den Noortgate, W., \& Goossens, L. (2019). Gender differences in loneliness across the lifespan: A meta-analysis. European Journal of Personality, 33, 642-654. https://doi.org/10.1002/per.2220

Majeno, A., Tsai, K. M., Huynh, V. W., McCreath, H., \& Fuligni, A. J. (2018). Discrimination and sleep difficulties during adolescence: The mediating roles of loneliness and perceived stress. Journal of Youth and Adolescence, 47, 135-147. https://doi.org/10.1007/ s10964-017-0755-8

Matthews, T., Danese, A., Caspi, A., Fisher, H. L., Goldman-Mellor, S., Kepa, A., Moffitt, T. E., Odgers, C. L., \& Arseneault, L. (2019). Lonely young adults in modern Britain: Findings from an epidemiological cohort study. Psychological Medicine, 49, 268-277. https://doi.org/10.1017/S0033291718000788

Matthews, T., Danese, A., Gregory, A. M., Caspi, A., Moffitt, T. E., \& Arseneault, L. (2017). Sleeping with one eye open: Loneliness and sleep quality in young adults. Psychological Medicine, 47, 2177-2186. https://doi.org/10.1017/S0033291717000629

Matthews, T., Danese, A., Wertz, J., Odgers, C. L., Ambler, A., Moffitt, T. E., \& Arseneault, L. (2016). Social isolation, loneliness and depression in young adulthood: A behavioural genetic analysis. Social Psychiatry and Psychiatric Epidemiology, 51, 339-348. https://doi.org/10.1007/s00127-016-1178-7

Morean, M. E., Krishnan-Sarin, S., Sussman, S., Foulds, J., Fishbein, H., Grana, R., \& O’Malley, S. S. (2019). Psychometric evaluation of the E-cigarette dependence scale. Nicotine \& Tobacco Research, 21, 1556-1564. https://doi.org/10.1093/ntr/ntx271

National Academies of Sciences, Engineering, and Medicine,. (2019). The Promise of Adolescence: Realizing Opportunity for All Youth. The National Academies Press. https://doi.org/10.17226/25388

Owens, J., Adolescent Sleep Working Group, \& Committee on Adolescence. (2014). Insufficient sleep in adolescents and young adults: 
An update on causes and consequences. Pediatrics, 134, e921e932. https://doi.org/10.1542/peds.2014-1696

Pasch, K. E., Laska, M. N., Lytle, L. A., \& Moe, S. G. (2010). Adolescent sleep, risk behaviors, and depressive symptoms: Are they linked? American Journal of Health Behavior, 34, 237-248. https://doi.org/10.5993/ajhb.34.2.11

Patrick, M. E., Bray, B. C., \& Berglund, P. A. (2016). Reasons for marijuana use among young adults and long-term associations with marijuana use and problems. Journal of Studies on Alcohol and Drugs, 77, 881-888. https://doi.org/10.15288/jsad.2016.77.881

Peltzer, K., \& Pengpid, S. (2017). Loneliness: Its correlates and associations with health risk behaviours among university students in 25 countries. Journal of Psychology in Africa, 27, 247-255. https://doi.org/10.1080/14330237.2017.1321851

Petitte, T., Mallow, J., Barnes, E., Petrone, A., Barr, T., \& Theeke, L. (2015). A systematic review of loneliness and common chronic physical conditions in adults. Open Psychology Journal, 8, 113132. https://doi.org/10.2174/1874350101508010113

Read, J. P., Merrill, J. E., Kahler, C. W., \& Strong, D. R. (2007). Predicting functional outcomes among college drinkers: Reliability and predictive validity of the Young Adult Alcohol Consequences Questionnaire. Addictive Behaviors, 32, 2597-2610. https://doi. org/10.1016/j.addbeh.2007.06.021

Rhew, I. C., Cadigan, J. M., \& Lee, C. M. (2021). Marijuana, but not alcohol, use frequency associated with greater loneliness, psychological distress, and less flourishing among young adults. Drug and Alcohol Dependence, 218, 108404. https://doi.org/10.1016/j. drugalcdep.2020.108404

Richard, A., Rohrmann, S., Vandeleur, C. L., Schmid, M., Barth, J., \& Eichholzer, M. (2017). Loneliness is adversely associated with physical and mental health and lifestyle factors: Results from a Swiss national survey. PLoS ONE, 12, e0181442. https://doi.org/ 10.1371/journal.pone.0181442

Segrin, C., \& Passalacqua, S. A. (2010). Functions of loneliness, social support, health behaviors, and stress in association with poor health. Health Communication, 25, 312-322. https://doi.org/10. 1080/10410231003773334

Shadel, W. G., Edelen, M. O., Tucker, J. S., Stucky, B. D., Hansen, M., \& Cai, L. (2014). Development of the PROMIS nicotine dependence item banks. Nicotine \& Tobacco Research, 16, S190-201. https://doi.org/10.1093/ntr/ntu032

Shovestul, B., Han, J., Germine, L., \& Dodell-Feder, D. (2020). Risk factors for loneliness: The high relative importance of age versus other factors. PLOS ONE, 15, e0229087. https://doi.org/10.1371/ journal.pone.0229087

Simons, J. S., Dvorak, R. D., Merrill, J. E., \& Read, J. P. (2012). Dimensions and severity of marijuana consequences: Development and validation of the Marijuana Consequences Questionnaire (MACQ). Addictive Behaviors, 37, 613-621. https://doi.org/10. 1016/j.addbeh.2012.01.008

Smith, K. J., Gavey, S., NE, R. I., Kontari, P., \& Victor, C. (2020). The association between loneliness, social isolation and inflammation: A systematic review and meta-analysis. Neuroscience \& Biobehavioral Reviews, 112, 519-541. https://doi.org/10.1016/j. neubiorev.2020.02.002

Sokol, R., Ennett, S., Gottfredson, N., \& Halpern, C. (2017). Variability in self-rated health trajectories from adolescence to young adulthood by demographic factors. Preventive Medicine, 105, 73-76. https://doi.org/10.1016/j.ypmed.2017.08.015
The GenIUSS Group. (2014). Best Practices for Asking Questions to Identify Transgender and Other Gender Minority Respondents on Population-Based Surveys. The Williams Institute. https://williamsinstitute.law.ucla.edu/publications/geniu ss-trans-pop-based-survey/.

Thompson, K., Roemer, A., \& Leadbeater, B. (2015). Impulsive personality, parental monitoring, and alcohol outcomes from adolescence through young adulthood. Journal of Adolescent Health, 57, 320-326. https://doi.org/10.1016/j.jadohealth.2015.05.005

Troxel, W. M., Rodriguez, A., Seelam, R., Tucker, J. S., Shih, R. A., \& D'Amico, E. J. (2019). Associations of longitudinal sleep trajectories with risky sexual behavior during late adolescence. Health Psychology, 38, 716-726. https://doi.org/10.1037/hea0000753

Troxel, W. M., Shih, R. A., Ewing, B., Tucker, J. S., Nugroho, A., \& D'Amico, E. J. (2017). Examination of neighborhood disadvantage and sleep in a multi-ethnic cohort of adolescents. Health \& Place, 45, 39-45. https://doi.org/10.1016/j.healthplace.2017. 03.002

Tucker, J. S., Orlando, M., \& Ellickson, P. L. (2003). Patterns and correlates of binge drinking trajectories from early adolescence to young adulthood. Health Psychology, 22, 79-87. https://doi.org/ 10.1037//0278-6133.22.1.79

US Census Bureau. (2018). Families \& living arrangements. https:// www.census.gov/topics/families.html.

VanderWeele, T. J., Hawkley, L. C., Thisted, R. A., \& Cacioppo, J. T. (2011). A marginal structural model analysis for loneliness: Implications for intervention trials and clinical practice. Journal of Consulting and Clinical Psychology, 79, 225-235. https://doi. org/10.1037/a0022610

von Soest, T., Luhmann, M., \& Gerstorf, D. (2020). The development of loneliness through adolescence and young adulthood: Its nature, correlates, and midlife outcomes. Developmental Psychology, 56, 1919-1934. https://doi.org/10.1037/dev0001102

Ware, J., Jr., Kosinski, M., \& Keller, S. D. (1996). A 12-Item ShortForm Health Survey: Construction of scales and preliminary tests of reliability and validity. Medical Care, 34, 220-233. https://doi. org/10.1097/00005650-199603000-00003

Wolfson, A. R., Carskadon, M. A., Acebo, C., Seifer, R., Fallone, G., Labyak, S. E., \& Martin, J. L. (2003). Evidence for the validity of a sleep habits survey for adolescents. Sleep, 26, 213-216. https:// doi.org/10.1093/sleep/26.2.213

Wu, S., Wang, R., Zhao, Y., Ma, X., Wu, M., Yan, X., \& He, J. (2013). The relationship between self-rated health and objective health status: A population-based study. BMC Public Health, 13, 320. https://doi.org/10.1186/1471-2458-13-320

Zhaoyang, R., Sliwinski, M. J., Martire, L. M., \& Smyth, J. M. (2019). Social interactions and physical symptoms in daily life: Quality matters for older adults, quantity matters for younger adults. Psychology \& Health, 34, 867-885. https://doi.org/10.1080/08870 446.2019.1579908

Publisher's Note Springer Nature remains neutral with regard to jurisdictional claims in published maps and institutional affiliations. 